Ann. Génét. Sél. anim., I973, 5 (I), 53-72.

\title{
CARACTÉRISTIQUES DÉMOGRAPHIQUES DE LA RACE OVINE SARDE
}

\author{
J.-J. LAUVERGNE, J. G. BOYAZOGLU *, R. CARTA ** et S. CASU ** \\ avec la collaboration technique de P. Carta, G. Picinelli, G. Ruda et L. Sanna
}

\author{
Laboratoive de Génétique factorielle, \\ Centre national de Recherches zootechniques, I. N. R. A., \\ 78350 Jouy en Josas \\ * Animal and Dairy Science Research Institute, \\ Pretoria, Rep. Sud-Africaine \\ ** Istituto Zootecnico e Caseario per la Sardegna, \\ Sassari, Sardaigne, Italie
}

\section{RÉSUMÉ}

La presque totalité des troupeaux inscrits au Flock-book de la race ovine sarde en Sardaigne a été analysée démographiquement ( 137 troupeaux sur I6I). On a pu les comparer à un échantillon de 140 troupeaux non inscrits.

La distribution des troupeaux inscrits et non inscrits selon le nombre des femelles obéit à une loi logarithmico-normale.

En moyenne, les troupeaux non inscrits comptent deux fois plus de brebis que les troupeaux inscrits ( 157 contre 85 ).

Le nombre moyen de femelles par bélier dont la descendance est susceptible d'être élevée est deux fois plus grand dans les troupeaux inscrits que dans les troupeaux non inscrits (73 femelles dans un cas, 32 dans l'autre).

L'intervalle de génération estimé par l'âge moyen des reproducteurs à la naissance de leurs progéniture est de 3,I an pour les mâles et de 4, 5 pour les femelles dans les troupeaux inscrits, les sexes des descendants étant groupés. Une comparaison avec les troupeaux non inscrits basée sur les animaux âgés de moins de trois ans fait apparaître une grande similitude pour les femelles. Quant aux mâles des troupeaux non inscrits, ils auraient un intervalle de génération un peu réduit.

Le taux de renouvellement annuel des femelles est de 17 p. Ioo dans les troupeaux inscrits, de $20 \mathrm{p}$. Ioo dans les troupeaux non inscrits.

Une étude de la migration des reproducteurs mâles révèle que la population sarde présente actuellement une structure pyramidale de sélection avec, au sommet, le centre de Barumini, fournisseur de la majeure partie des béliers étrangers utilisés dans les troupeaux inscrits, mais ne recevant, par contre, aucun gène de l'extérieur. Une telle situation hiérarchisée, bien qu'assez généralement observée au cours de l'évolution des races modernes de bétail, devrait être transitoire, si l'on veut augmenter l'efficacité de la sélection en élargissant ses bases. 


\section{INTRODUCTION}

L'élevage ovin laitier est implanté depuis très longtemps en Sardaigne. D'aspect traditionnel, extensif ou semi-extensif, il est surtout basé sur 1'utilisation des pâturages naturels qui représentent plus de I 500 ooo ha dans 1'île (CASU, I97I). Le lait trait est, dans sa quasi-totalité, utilisé pour la production fromagère et la plus grande partie de ce fromage, de pâte dure (Pecorino Romano et Fiore Sardo), est destinée à l'exportation (IDDA, I969).

La population ovine estimée déjà à I Ioo 000 têtes au début du XVII ${ }^{\mathbf{e}}$ siècle (CARILlo cité par Passino, I936), tout en subissant des fluctuations importantes, présente ces cinquante dernières années une tendance générale à l'accroissement. On comptait 2 millions d'animaux en I9I8 (PAssino, I936) ; actuellement le chiffre de 2500000 serait atteint (CASU, I97I).

Après s'être soucié, dès la fin du siècle dernier, d'améliorer la qualité et d'adapter le type du produit fini au goût des acheteurs étrangers, on a voulu également augmenter la productivité du troupeau laitier. Compte tenu des précédents échecs de croisements avec des races exotiques, on s'est finalement orienté vers l'amélioration des aptitudes laitières de la race autochtone, bien adaptée aux conditions difficiles du milieu local (PAssino, r936). Toutefois, comme le note cet auteur, les progrès sont lents et ce n'est qu'en I927 qu'apparaît le Flock-book de la race sarde qui inscrit cette année-là les animaux de 7 élevages de la province de Cagliari. L'activité de cet organisme s'étendra aux deux autres provinces à partir de I933.

De nombreuses études ont été consacrées à l'amélioration de la brebis sarde, entre autres : BoNELLI (I955, I962), BETTINI (I952). Jusqu'à présent, cependant, l'analyse démographique du troupeau avait été un peu négligée. Plusieurs considérations montrent néanmoins l'utilité de telles connaissances, aussi bien pour la solution de problèmes théoriques, comme l'étude des fréquences géniques (CASU et al., I970), que pour celle de problèmes pratiques, comme la mise en place d'un plan de sélection rationnel.

En I970, une enquête préliminaire a été entreprise pour combler en partie cette lacune. Elle avait pour objet essentiel d'estimer les quelques paramètres de base (taille de troupeau, taux de renouvellement) et de préciser la migration des mâles qui, constituant le sexe le moins fréquent, jouent un rôle génétique très important. Le présent article rassemble les résultats de cette enquête.

\section{MATÉRIEL ET MÉTHODES}

Si l'on excepte les 4 troupeaux expérimentaux de l'Istituto Zootecnico e Caseario de Sassari, la population ovine sarde est répartie dans des troupeaux commerciaux inscrits et non inscrits. Il y a I6I troupeaux inscrits pour toute l'île, alors que le nombre des troupeaux non inscrits oscille autour de I 5 ooo (il n'existe malheureusement pas de statistiques précises sur ces derniers).

Notre enquête, menée au cours des mois de juin et de juillet 1970, a porté en principe sur tous les troupeaux inscrits et sur un échantillon de même taille de troupeaux non inscrits. 

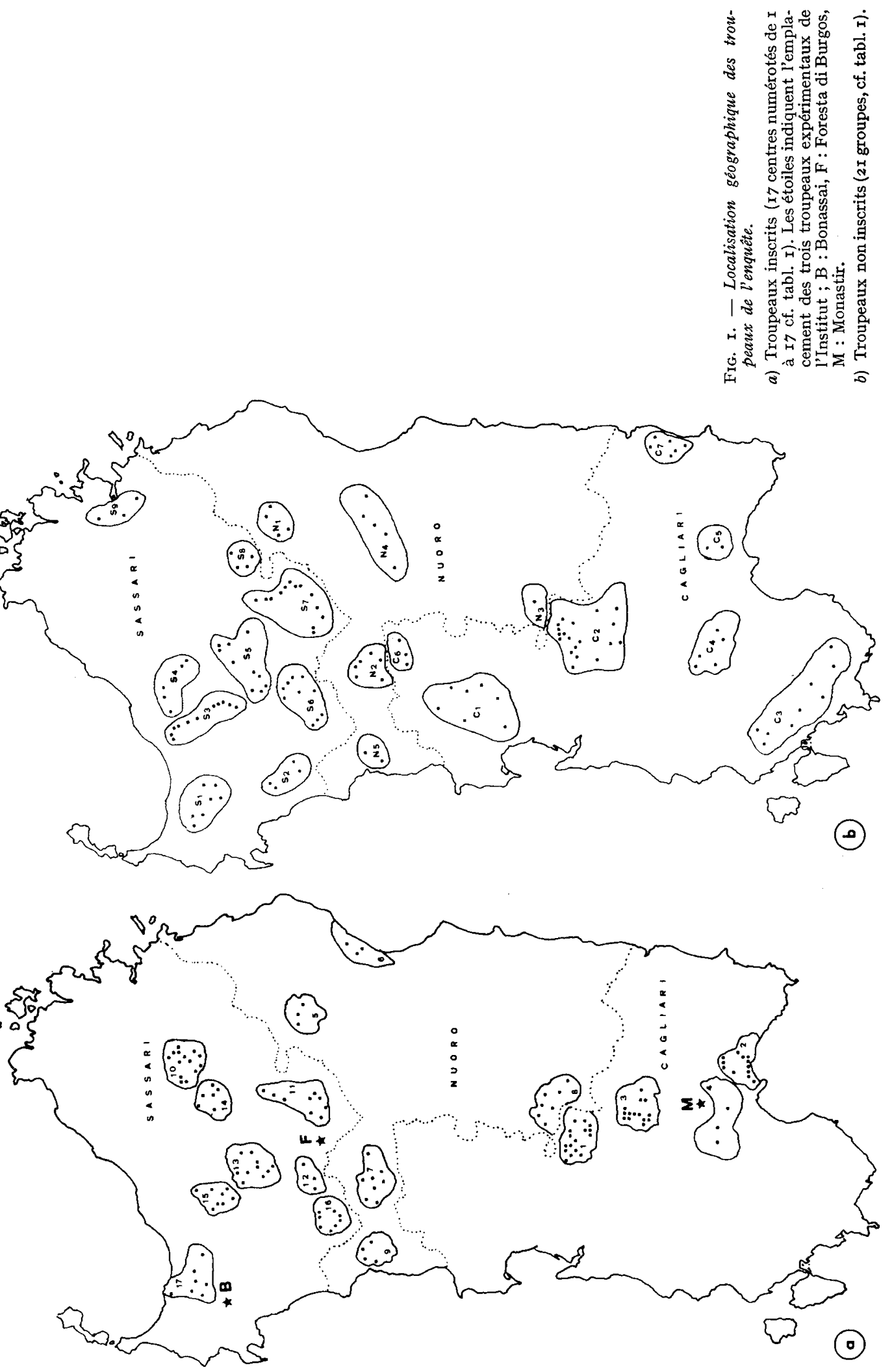
Troupeaux inscrits et

Localisation géograph des reproductrices et reproduc

\begin{tabular}{|c|c|c|c|c|c|c|c|}
\hline \multirow{3}{*}{ Province } & \multirow{3}{*}{ Localité } & & & \multicolumn{4}{|c|}{ Troupeaux inscrits } \\
\hline & & Numéros & Nombre & $\begin{array}{r}\text { Effectif } \\
\text { repr }\end{array}$ & $\begin{array}{l}\text { des femelles } \\
\text { ductrices }\end{array}$ & $\begin{array}{r}\text { Effect } \\
\text { rep }\end{array}$ & $\begin{array}{l}\text { des mâles } \\
\text { ducteurs }\end{array}$ \\
\hline & & centres & troupeaux & total & $\left|\begin{array}{c}\text { moyen } \\
\text { par troupeau }\end{array}\right|$ & total & $\begin{array}{r}\text { moyer } \\
\text { par troup }\end{array}$ \\
\hline Cagliari & $\begin{array}{l}\text { Barumini } \\
\text { Guasila } \\
\text { Iglesias } \\
\text { Monastir } \\
\text { Muravera } \\
\text { Oristano } \\
\text { Quartu } \\
\text { Sedilo }\end{array}$ & $\begin{array}{l}1 \\
3 \\
4\end{array}$ & $\begin{array}{l}16 \\
15 \\
\\
4\end{array}$ & $\begin{array}{r}1536 \\
1379 \\
\quad 457 \\
1100\end{array}$ & $\begin{array}{r}96,0 \\
91,9 \\
114,2 \\
91,7\end{array}$ & $\begin{array}{r}17 \\
14 \\
5\end{array}$ & $\begin{array}{l}1,06 \\
0,93 \\
1,25 \\
1,16\end{array}$ \\
\hline Nuoro & $\begin{array}{l}\text { Bitti } \\
\text { Borore } \\
\text { Dorgali } \\
\text { Flussio } \\
\text { Macomer } \\
\text { Nuragus } \\
\text { Oliena } \\
\text { Suni }\end{array}$ & $\begin{array}{l}5 \\
6 \\
7 \\
8 \\
9 \\
9\end{array}$ & $\begin{array}{l}3 \\
3 \\
\\
8 \\
7 \\
4\end{array}$ & $\begin{array}{l}230 \\
366 \\
647 \\
526 \\
340\end{array}$ & $\begin{array}{r}76,7 \\
122,0 \\
80,9 \\
75,1 \\
85,0\end{array}$ & $\begin{array}{l}3 \\
3 \\
3 \\
9 \\
8 \\
4\end{array}$ & $\begin{array}{l}1,00 \\
1,00 \\
1,12 \\
1,14 \\
1,00\end{array}$ \\
\hline Sassari & $\begin{array}{l}\text { Berchidda } \\
\text { Bono } \\
\text { Bonorva } \\
\text { Budduso } \\
\text { Mores } \\
\text { Nulvi } \\
\text { Olbia } \\
\text { Oschiri } \\
\text { Osilo } \\
\text { Ploaghe } \\
\text { Pozzo-Maggiore } \\
\text { Sassari } \\
\text { Villanova }\end{array}$ & $\begin{array}{l}10 \\
11 \\
12 \\
13 \\
\\
\\
14 \\
\\
15 \\
15 \\
17 \\
17\end{array}$ & $\begin{array}{r}14 \\
9 \\
3 \\
\\
10\end{array}$ & $\begin{array}{r}1669 \\
410 \\
148 \\
\\
985 \\
\\
\\
555 \\
\\
550 \\
371 \\
371\end{array}$ & $\begin{array}{r}119,2 \\
45,6 \\
49,3 \\
98,5\end{array}$ & $\begin{array}{r}22 \\
9 \\
3 \\
\\
15\end{array}$ & $\begin{array}{l}1,57 \\
1,00 \\
1,00 \\
1,50 \\
\\
1,33 \\
\\
1,37 \\
1,00 \\
0,85\end{array}$ \\
\hline $\begin{array}{l}\text { Cagliari } \\
\text { Nuoro } \\
\text { Sassari }\end{array}$ & & & $\begin{array}{l}47 \\
25 \\
65\end{array}$ & $\begin{array}{l}4 \quad 472 \\
2109 \\
5089\end{array}$ & $\begin{array}{l}95,2 \\
84,4 \\
78,3\end{array}$ & $\begin{array}{l}50 \\
27 \\
82\end{array}$ & $\begin{array}{l}1,06 \\
1,08 \\
1,26\end{array}$ \\
\hline Sardaigne & & & 137 & 11670 & 85,2 & 159 & 1,16 \\
\hline
\end{tabular}




\section{rits de l'enquête}

statistique globale

sents en juin-juillet $197^{\circ}$

\begin{tabular}{|c|c|c|c|c|c|c|c|}
\hline \multirow{3}{*}{$\begin{array}{l}\text { Nombre } \\
\text { moyen } \\
\text { e femelles } \\
\text { par bélier }\end{array}$} & \multicolumn{7}{|c|}{ Troupeaux non inscrits } \\
\hline & \multirow{2}{*}{$\begin{array}{l}\text { Numéros } \\
\text { des } \\
\text { groupes }\end{array}$} & \multirow{2}{*}{$\begin{array}{c}\text { Nombre } \\
\text { de } \\
\text { troupeaux }\end{array}$} & \multicolumn{2}{|c|}{$\begin{array}{l}\text { Effectif des femelles } \\
\text { reproductrices }\end{array}$} & \multicolumn{2}{|c|}{$\begin{array}{l}\text { Effectif des mâles } \\
\text { reproducteurs }\end{array}$} & \multirow{2}{*}{$\begin{array}{c}\text { Nombre } \\
\text { moyen } \\
\text { de femelles } \\
\text { par bélier }\end{array}$} \\
\hline & & & total & moyen & total & moyen & \\
\hline \multirow{2}{*}{$\begin{array}{l}90,4 \\
98,5\end{array}$} & $\mathrm{C}_{2}$ & 17 & 1914 & 112,9 & 48 & 2,8 & 39,9 \\
\hline & $\mathrm{C}_{3}$ & 9 & 2007 & 223,0 & 51 & 5,7 & 39,4 \\
\hline \multirow[t]{3}{*}{91,4} & $\mathrm{C}_{4}$ & 9 & 2279 & 253,2 & 55 & 6,1 & 41,4 \\
\hline & $\mathrm{C}_{1}$ & 6 & 777 & 129,5 & 32 & 5,3 & 24,3 \\
\hline & $\mathrm{C}_{1}$ & 7 & 792 & 113,1 & 23 & 3,3 & 34,4 \\
\hline \multirow[t]{2}{*}{78,6} & $\mathrm{C}_{5}$ & 4 & 842 & 210,5 & 18 & 4,5 & 46,8 \\
\hline & $\mathrm{C}_{6}$ & 3 & 393 & 131,0 & 10 & 3,3 & 39,3 \\
\hline \multirow[t]{2}{*}{76,7} & $N_{1}$ & 4 & 1217 & 306,8 & 28 & 7,0 & 43,5 \\
\hline & $\mathrm{N}_{2}$ & 6 & 1009 & 168,2 & 21 & 3,5 & 48,1 \\
\hline 122,0 & $\mathrm{~N}_{5}$ & 2 & 194 & 97,0 & 2 & 1,0 & 97,0 \\
\hline $\begin{array}{l}71,9 \\
65,8\end{array}$ & $\mathrm{~N}_{3}$ & 1 & 145 & 145,0 & 3 & 3,0 & 48,3 \\
\hline & $\mathrm{N}_{4}$ & 5 & 1170 & 234,0 & 28 & 5,6 & 41,8 \\
\hline 85,0 & & & & & & & \\
\hline \multirow{4}{*}{$\begin{array}{l}75,9 \\
45,6 \\
49,3\end{array}$} & & & & & & & \\
\hline & $\mathrm{S}_{7}$ & 13 & 1886 & 145,1 & 41 & 3,2 & 46,0 \\
\hline & $\mathrm{S}_{6}$ & 10 & 1653 & 165,3 & 56 & 5,6 & 29,5 \\
\hline & $\mathrm{S}_{8}$ & 4 & 665 & 166,3 & 17 & 4,3 & 39,1 \\
\hline \multirow[t]{3}{*}{65,7} & $\mathrm{~S}_{5}$ & 10 & 1553 & 155,3 & 30 & 3,0 & 51,8 \\
\hline & $S_{4}$ & 5 & 599 & 119,8 & 24 & 4,8 & 24,9 \\
\hline & $\mathrm{S}_{9}$ & 3 & 368 & 122,7 & 7 & 2,3 & 52,6 \\
\hline 69,4 & $\mathrm{~S}_{3}$ & 11 & 889 & 80,8 & 44 & 4,0 & 20,2 \\
\hline $\begin{array}{l}50,0 \\
46,4\end{array}$ & & & & & & & \\
\hline \multirow[t]{2}{*}{61,8} & $\mathrm{~S}_{1}$ & 7 & 965 & 137,9 & 41 & 5,8 & 23,5 \\
\hline & $\mathrm{S}_{2}$ & 4 & 606 & 151,5 & 27 & 6,8 & 22,4 \\
\hline 89,0 & & 55 & 9004 & 163,7 & 319 & 5,8 & 28,2 \\
\hline 78,1 & & 18 & 3735 & 207,5 & 82 & 4,6 & 45,5 \\
\hline 62,0 & & 67 & 9184 & 137,7 & 287 & 4,3 & 32,0 \\
\hline 73,3 & & 140 & 21923 & 156,6 & 688 & 4,9 & 31,9 \\
\hline
\end{tabular}


L'organisation des contrôles d'aptitude pour les troupeaux inscrits est basée sur un regroupement de ces derniers en I 7 centres. Pour diverses causes (absence ou refus du berger, difficultés d'accès, etc.), un certain nombre de troupeaux inscrits n'ont pu être inventoriés : 2 dans la province de Cagliari, I 5 à Nuoro et 7 à Sassari : au total, I 37 troupeaux ont été visités sur I6I (86 p. I00). La figure $x, a$ donne la localisation géographique des troupeaux inscrits dans l'île et l'on trouvera dans le tableau I la liste des centres numérotés de I à I 7 .

L'échantillon des troupeaux non inscrits a été choisi au départ de manière à couvrir les principaux milieux pastoraux, compte tenu de la densité du cheptel et de la taille des troupeaux. Les difficultés rencontrées localement, généralement plus grandes que pour les troupeaux inscrits, ont néanmoins conduit à modifier quelque peu le plan d'échantillonnage initial (I 40 troupeaux finalement retenus). On s'est efforcé cependant de respecter, autant que possible, les principes énoncés plus haut. D'après leur situation géographique, les troupeaux de l'échantillon ont été répartis arbitrairement en $2 \mathrm{I}$ groupes qui portent le nom de la localité la plus proche et dont la liste est donnée dans le tableau I. Ils sont numérotés de $C_{1}$ à $C_{7}$, de $N_{1}$ à $N_{5}$ et de $S_{1}$ à $S_{9}$, selon l'initiatiale de la province. Leur localisation est donnée dans la figure $\mathbf{I}, b$.

Dans chaque troupeau inscrit, l'enquêteur a dénombré les animaux reproducteurs mâles et femelles présents et les agnelles, en notant toujours l'âge. Au moment de l'enquête, qui avait lieu pendant la période de lutte, on a également noté l'origine des béliers utilisés.

Pour les troupeaux non inscrits, on s'est efforcé de réunir les mêmes renseignements, mais avec moins de succès. Le relevé de l'âge a dû être fait empiriquement d'après la denture et son imprécision, au-delà de 3 ans notamment, est grande. Quant à l'origine des béliers employés, les statistiques manquent, là aussi, de précision.

Outre la présentation des tableaux de dénombrement et des graphiques qui illustrent et résument chaque point de l'enquête, nous avons cherché à déterminer la forme mathématique de certaines distributions par la droite de Henry et utilisé le test $t$ pour comparer les moyennes et les fréquences.

\section{RÉSULTATS}

\section{A. - Effectifs et distribution par sexe et par âge}

Le tableau I présente à la fois la liste des centres, le nombre des troupeaux inscrits et non inscrits dans leurs centres respectifs, les effectifs de femelles reproductrices, sans compter les agnelles (à cause de leur agnelage tardif, on n'élève jamais leurs produits), ceux des mâles reproducteurs ainsi que la proportion de femelles par mâle reproducteur.

\section{Forme mathématique de la distribution des troupeaux selon leur taille.}

Comme on peut le voir sur la figure 2 ( $a$ et $b$ ), les distributions des troupeaux selon l'effectif de brebis sont fortement dissymétriques.

L'ajustement à une loi logarithmico-normale donne satisfaction, comme semblent l'indiquer les droites de Henry de la figure 3.

\section{Effectifs des mâles, nombre de femelles par mâle.}

86,8 p. Ioo des 137 troupeaux inscrits inspectés utilisent, quelle que soit leur taille, un seul bélier pendant l'époque de monte contrôlée qui dure de 40 à 50 jours. Le nombre moyen de béliers utilisés par troupeau non inscrit est de 5 environ.

Ainsi, pour la population inscrite que nous avons contrôlée, le rapport mâles/ femelles est de I/72 pendant la période lutte contrôlée, tandis que dans les troupeaux non inscrits le rapport est de $\mathrm{r} / 32$ pour toute la période de lutte. Les distributions d'effectifs des lots"de lutte sont présentées sur la figure 4 .

De la même manière que précédemment, on a testé graphiquement la lognormalité de ces deux distributions (cf. fig. 3). 

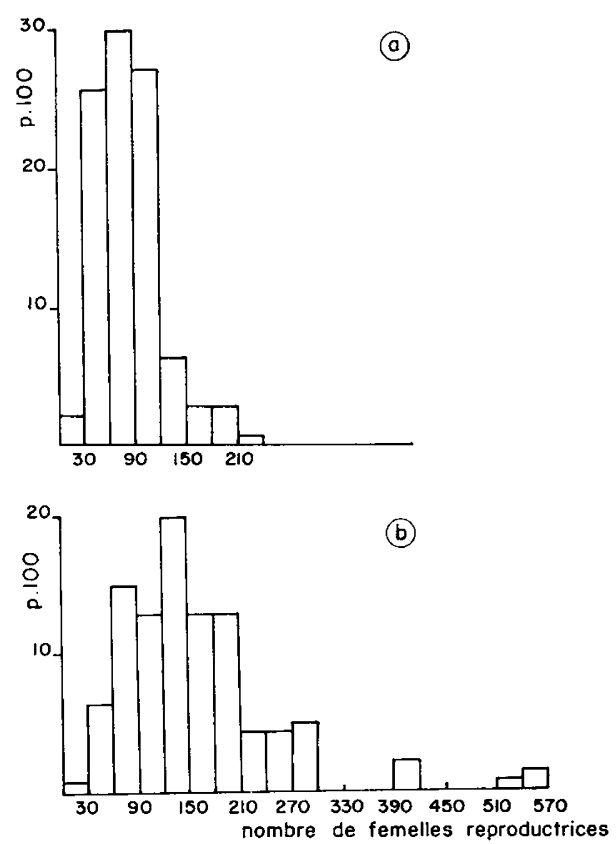

FIG. 2. - Distribution des troupeaux de l'enquête selon l'effectif des femelles

a) Troupeaux non inscrits.

b) Troupeaux inscrits.

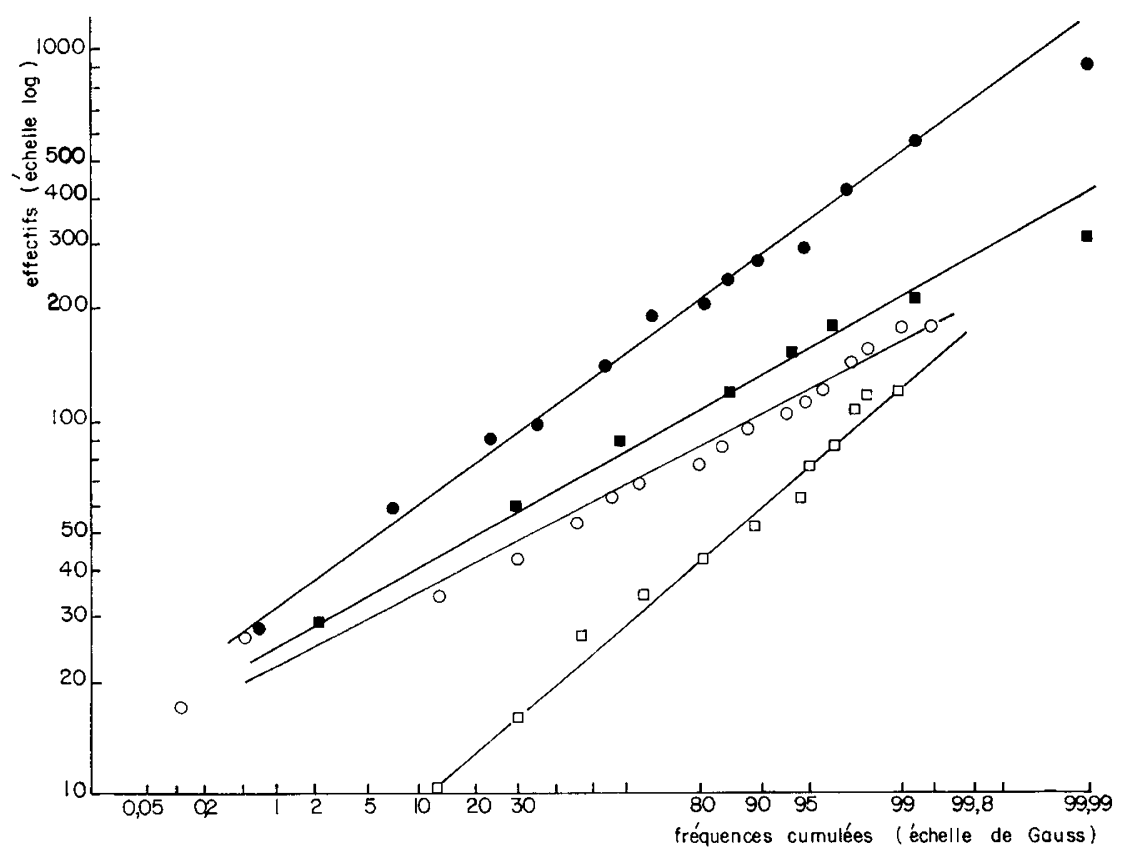

FIG. 3. - - Test de log-normalité des lois de distribution de la taille des troupeaux de brebis et du nombre moyen de femelles par bélier dont la descendance est susceptible d'être élevée

- Taille des troupeaux inscrits $(n=137)$

- Taille des troupeaux non inscrits $(n=140)$

$\square$ Nombre moyen de femelles par bélier, troupeaux inscrits

- Nombre moyen de femelles par bélier, troupeaux non inscrits 

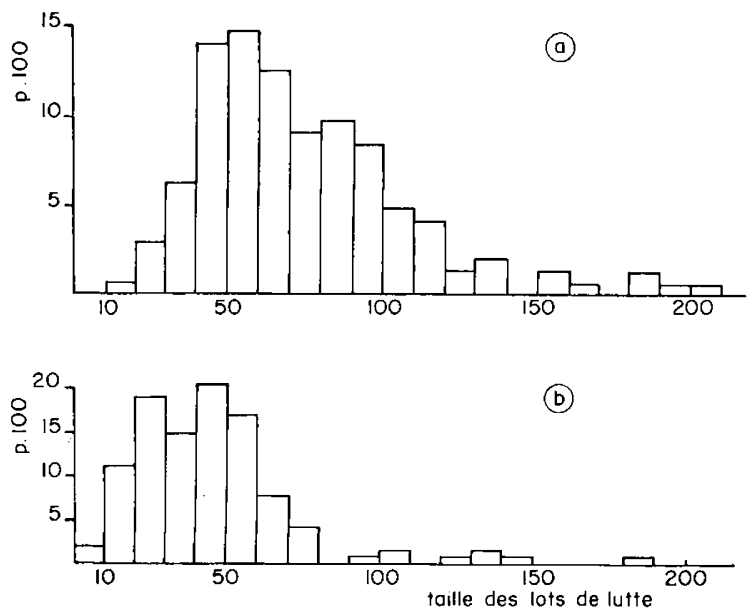

FIG. 4. - Distribution des effectifs de femelles par bélier dont la descendance est susceptible d'être élevée

a) Troupeaux inscrits.

b) Troupeaux non inscrits.

3. Répartition selon l'âge.

L'histogramme de la répartition des animaux mâles ou femelles selon leur âge est donné dans la figure 5 pour les troupeaux inscrits.

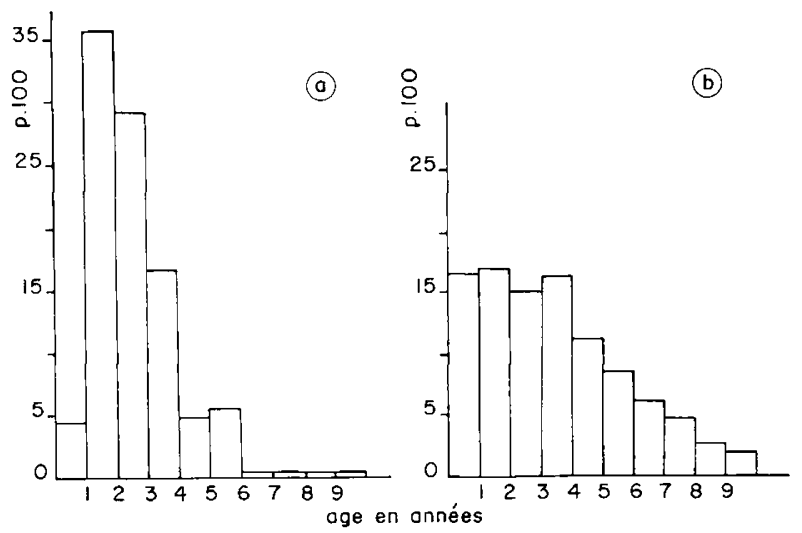

FIG. 5. - Distribution des reproducteurs selon leur âge dans les troupeaux inscrits

a) Les mâles.

b) Les femelles.

Pour les troupeaux non inscrits où la précision, au-delà de 3 ans, est faible, on a dû regrouper les données au-delà de cet âge. Elles sont présentées, ainsi que celles des troupeaux inscrits, dans le tableau 2. 
TABLEAU 2

Distribution des béliers et des brebis selon leur âge dans les troupeaux inscrits et non inscrits

\begin{tabular}{|c|c|c|c|c|c|c|c|c|c|c|c|}
\hline \multirow{2}{*}{$\begin{array}{c}\text { Type } \\
\text { de } \\
\text { troupeaux }\end{array}$} & \multirow{2}{*}{ Sexe } & \multicolumn{2}{|c|}{ de 0 à 1 an } & \multicolumn{2}{|c|}{ de 1 à 2 ans } & \multicolumn{2}{|c|}{ de 2 à 3 ans } & \multicolumn{2}{|c|}{3 ans et plus } & \multirow{2}{*}{$\begin{array}{l}\text { Nbre } \\
\text { total }\end{array}$} & \multirow{2}{*}{$\begin{array}{c}\text { Age } \\
\text { moyen }\end{array}$} \\
\hline & & N & $\%$ & $\mathrm{~N}$ & $\%$ & $\mathrm{~N}$ & $\%$ & $\mathrm{~N}$ & $\%$ & & \\
\hline Inscrits & $\begin{array}{l}\text { Béliers } \\
\text { Brebis }\end{array}$ & $\begin{array}{r}7 \\
2325\end{array}$ & $\begin{array}{r}4,4 \\
16,7\end{array}$ & $\begin{array}{r}57 \\
2394\end{array}$ & $\begin{array}{l}35,9 \\
17,2\end{array}$ & $\begin{array}{r}47 \\
2130\end{array}$ & $\begin{array}{l}29,6 \\
15,3\end{array}$ & $\begin{array}{r}48 \\
7070\end{array}$ & $\begin{array}{l}30,2 \\
50,8\end{array}$ & $\begin{array}{r}159 \\
13919\end{array}$ & $\begin{array}{l}3,1 \\
3,9\end{array}$ \\
\hline $\begin{array}{c}\text { Non } \\
\text { inscrits }\end{array}$ & $\begin{array}{l}\text { Béliers } \\
\text { Brebis }\end{array}$ & $\begin{array}{r}122 \\
5640\end{array}$ & $\begin{array}{l}22,8 \\
20,5\end{array}$ & $\begin{array}{r}131 \\
4031\end{array}$ & $\begin{array}{l}24,5 \\
14,6\end{array}$ & $\begin{array}{r}114 \\
+174\end{array}$ & $\begin{array}{l}21,4 \\
15,1\end{array}$ & $\begin{array}{r}167 \\
13718\end{array}$ & $\begin{array}{l}31,3 \\
49,8\end{array}$ & $\begin{array}{r}534 \\
27563\end{array}$ & \\
\hline
\end{tabular}

4. Comparaison des moyennes d'effectifs et d'âge.

Les valeurs moyennes des effectifs des femelles et des mâles dans les troupeaux inscrits ont été comparées à leurs valeurs dans les troupeaux non inscrits par le test $t$ que 1'on a appliqué également à la taille moyenne des lots de lutte et aux différentes fréquences dans les classes d’âge envisagées dans le tableau précédent. Les résultats de ces tests sont donnés dans le tableau 3.

\section{TABLEAU 3}

Comparaisons entre troupeaux inscrits et non inscrits portant sur les effectifs et les ages

\begin{tabular}{|c|c|c|c|c|c|}
\hline \multirow{2}{*}{ Grandeurs comparées } & \multicolumn{2}{|c|}{$\begin{array}{l}\text { Troupeaux } \\
\text { inscrits }\end{array}$} & \multicolumn{2}{|c|}{$\begin{array}{c}\text { Troupeaux } \\
\text { non inscrits }\end{array}$} & \multirow{2}{*}{$\begin{array}{c}\text { Valeur de } t \\
\left({ }^{2}\right)\end{array}$} \\
\hline & effectifs & $x$ & effectifs & $x$ & \\
\hline Nombre moyen de béliers par troupeau $\left(^{1}\right) \ldots$ & 137 & 1,2 & 140 & 4,9 & $10,52 * *$ \\
\hline Nombre moyen de femelles par troupeau .... & 137 & 85,2 & 140 & 156,6 & $3,58^{* *}$ \\
\hline Nombre moyen de femelles par bélier $\left({ }^{1}\right) \ldots$ & & 73,3 & & 31,9 & $6,77 * *$ \\
\hline Fréquence des béliers nés dans l'année .. & 159 & 0,044 & 534 & 0,228 & $7,53 * *$ \\
\hline Fréquence des béliers de 1 à 2 ans .... & 159 & 0,359 & 534 & 0,245 & $2,69 * *$ \\
\hline Fréquence des béliers de 2 à 3 ans ........ & 159 & 0,296 & 534 & 0,214 & $2,03^{*}$ \\
\hline Fréquence des béliers de plus de 3 ans $\ldots .$. & 159 & 0,302 & 534 & 0,313 & $0,27 \mathrm{NS}$ \\
\hline Fréquence des agnelles $\ldots \ldots \ldots \ldots \ldots \ldots$ & 13919 & 0,167 & 27563 & 0,205 & $9,53^{* *}$ \\
\hline Fréquence des brebis de 1 à 2 ans $\ldots$. & 13919 & 0,172 & 27563 & 0,146 & $6,77 * *$ \\
\hline Fréquence des brebis de 2 à 3 ans $\ldots \ldots$ & 13919 & 0,153 & 27563 & 0,151 & $0,54 \mathrm{NS}$ \\
\hline Fréquence des brebis de plus de 3 ans ..... & 13919 & 0,508 & 27563 & 0,498 & $1,92 \mathrm{NS}$ \\
\hline
\end{tabular}

(1) Il s'agit des béliers dont la descendance est susceptible d'être élevée.

(2) NS écart non significatif au seuil de 5 p. 100.

* écart significatif au seuil de 5 p. 100.

** écart significatif au seuil de 1 p. 100. 
J.-J. LAUVERGNE ET COLL.

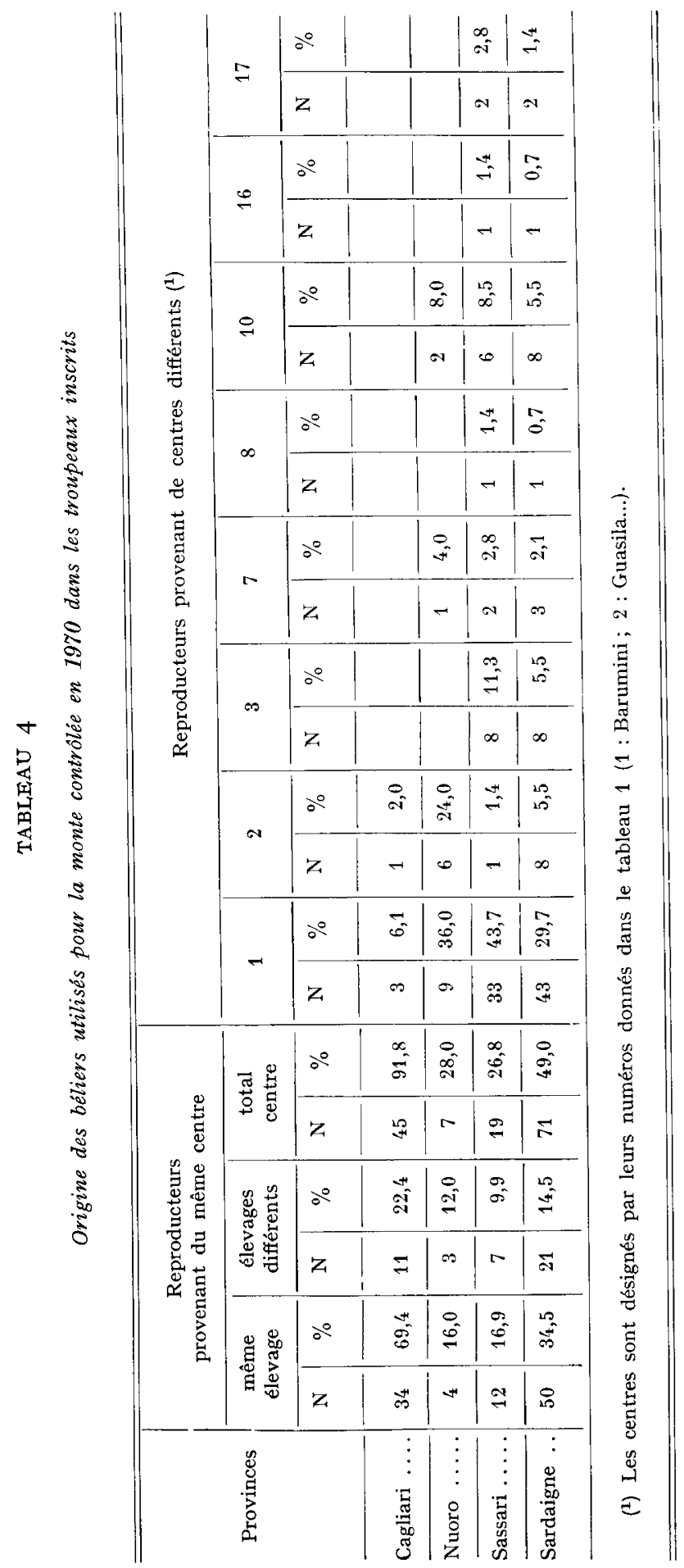


DÉMOGRAPHIE DU MOUTON “ SARDE 》

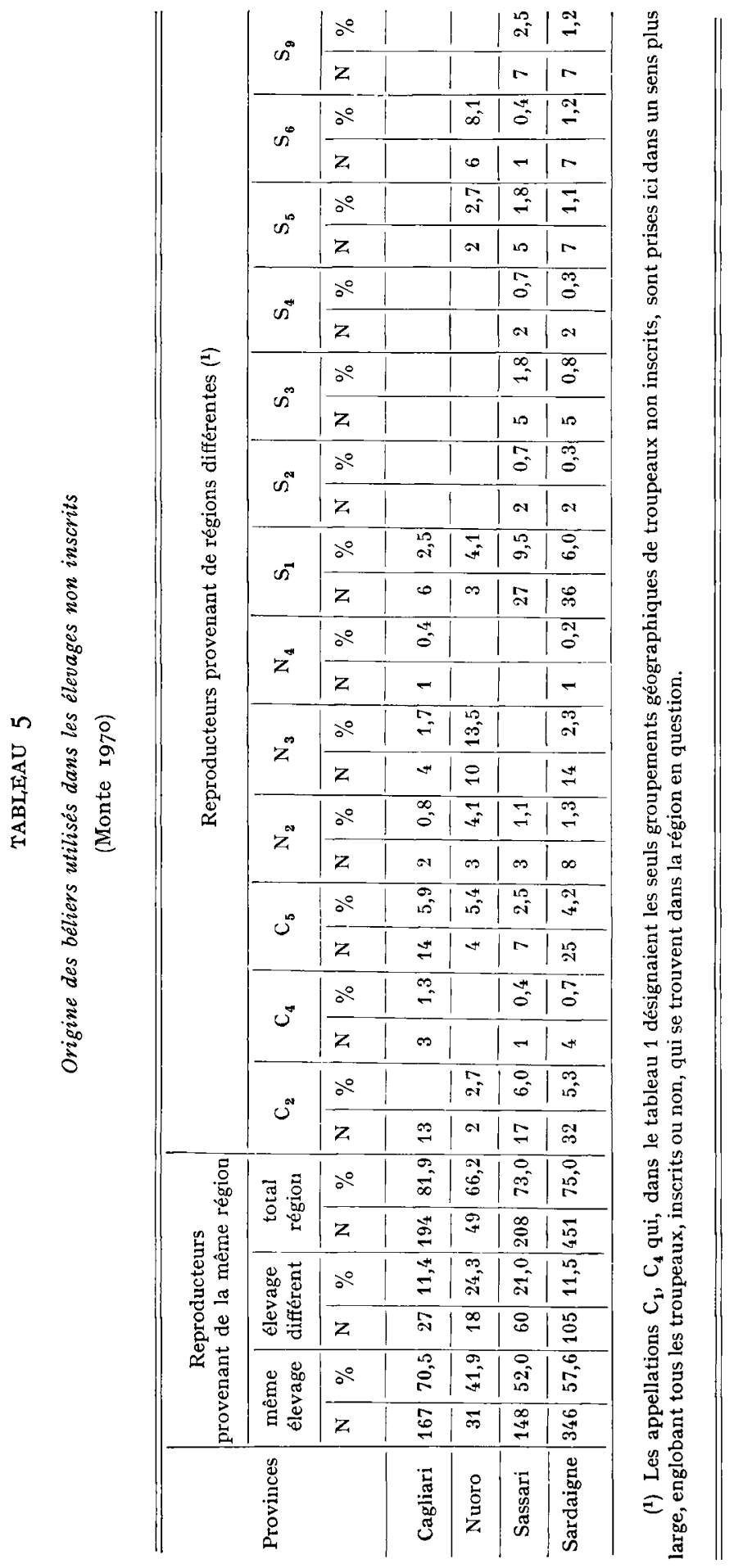



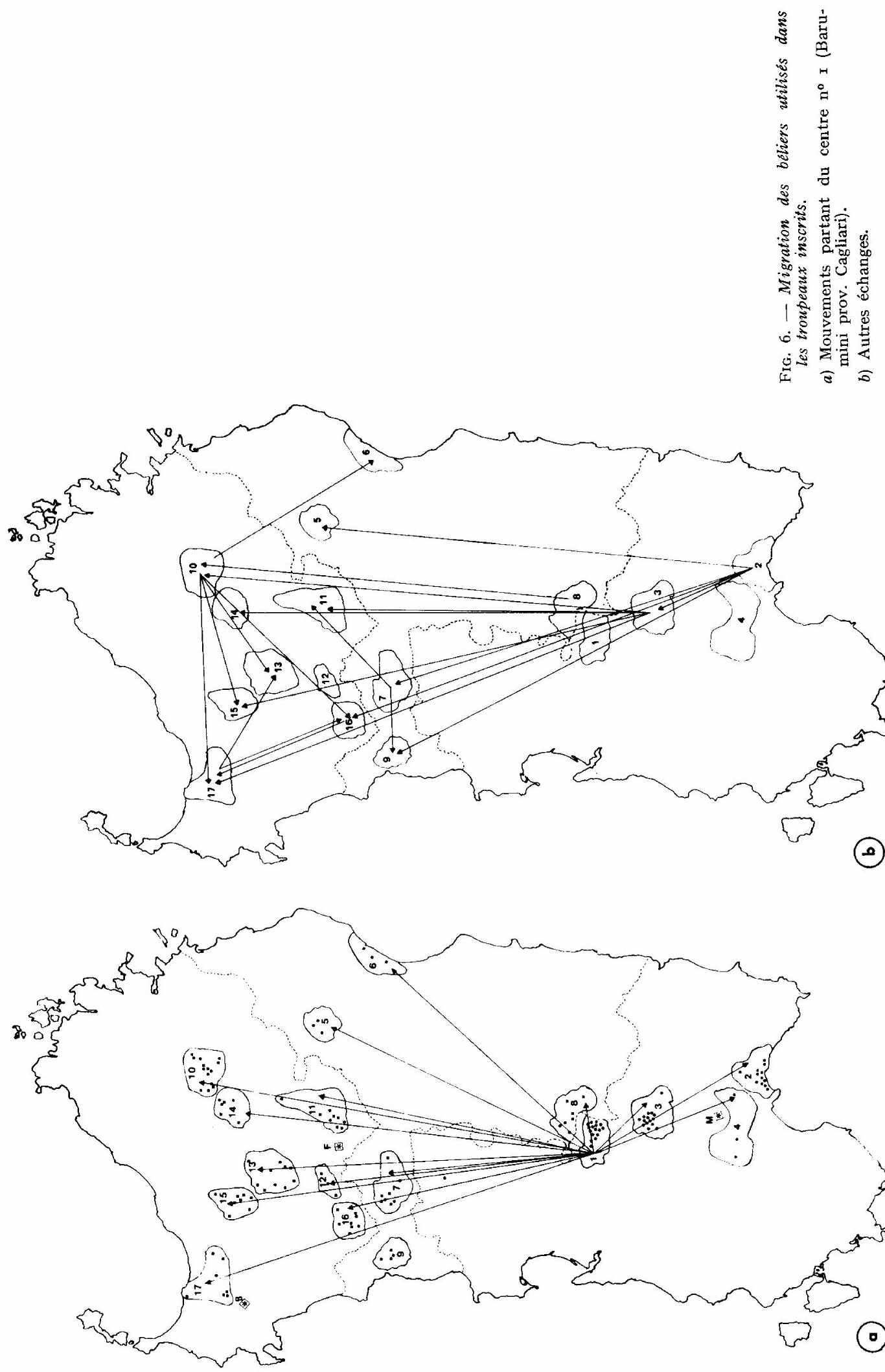


\section{B. - Migration des mâles}

Dans le tableau 4, on donne l'origine (centre) des mâles inscrits utilisés pour la monte " contrôlée " de I 970 dans les troupeaux inscrits.

Dans le tableau 5, on donne l'origine des mâles non inscrits ou inscrits utilisés en I970 dans les troupeaux non inscrits.

Les mouvements des reproducteurs sont illustrés par la figure $6(a$ et $b)$ pour les troupeaux inscrits. Par souci de clarté on a utilisé deux cartes : sur la première, sont notés les seuls mouvements en provenance du centre de Barumini, prov. Cagliari, dont l'importance semble primordiale; sur la seconde, les autres échanges.

Pour les troupeaux non inscrits on a également utilisé deux cartes (fig. 7 , $a$ et $b)$ : la première carte présente les échanges entre troupeaux non inscrits et la seconde les mouvements de béliers provenant de troupeaux inscrits.

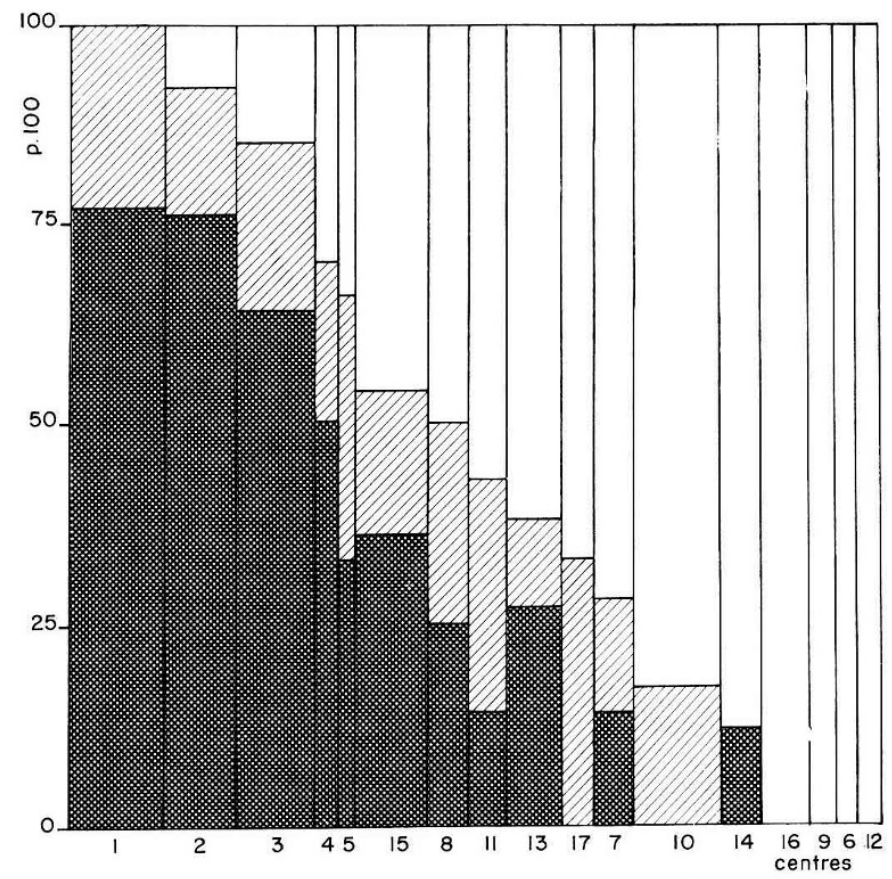

FIG. 8. - Répartition des béliers utilisés dans les troupeaux inscrits selon leur origine

- provenant du même troupeau dans le même centre (grisé),

- provenant d'un autre troupeau du même centre (hachuré),

- provenant d'un autre centre (blanc).

La largeur de la bande attribuée à chaque centre désigné ici par un numéro (cf. liste dans le tabl. I) est proportionnelle au nombre de reproducteurs mâles employés.

Sur la figure 8 , on a représenté les béliers utilisés dans les troupeaux inscrits selon leur fréquence, distinguant ceux qui proviennent du même troupeau, du même centre ou d'un autre centre. 


\section{DISCUSSION}

\section{A. - La loi de distribution des troupeaux selon leur taille}

Nous n'avons pas trouvé d'auteur qui se soit intéressé à la forme mathématique de la distribution des troupeaux de brebis selon leur taille. Certains chercheurs ont cependant publié des données permettant de faire les mêmes tests que nous ; ainsi WIENER (I96I) pour plusieurs races anglaises. Dans la figure 9, nous donnons les résultats d'ajustements des données de cet auteur à la loi log-normale. On voit qu'ils sont, dans l'ensemble, assez bons, venant ainsi corroborer les nôtres.

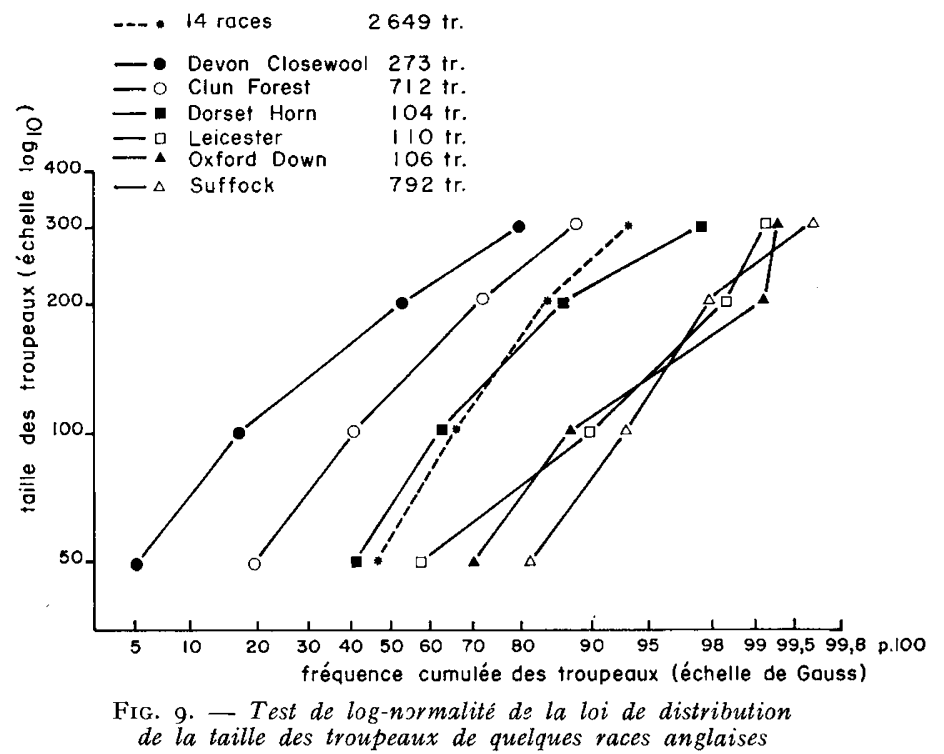

(Données de WIEner, I96I, tabl. I)

A tout réfléchir, on ne doit pas s'étonner que la taille des troupeaux de moutons, en rapport direct avec la superficie dont chaque éleveur dispose, obéisse à une telle loi puisque, précisément, on observe que la superficie des exploitations agricoles ( $\left.{ }^{(}\right)$, comme celle, plus générale, des revenus, suit une loi log-normale.

\section{B. - Taille moyenne des troupeaux inscrits et non inscrits}

La taille moyenne actuelle des troupeaux non inscrits - sensiblement égale à celle estimée par IDDA (r969) en I967 - est près du double de celle des troupeaux inscrits, la différence étant significative (tabl. 3). Cela est en partie, semble-t-il un,

(1) Cela est aisément vérifiable à partir des statistiques données par le Ministère de l'Agriculture en France, par exemple (Anonyme, r969). 
legs du passé, l'effort de sélection ayant débuté dans les régions de l'île à petite superficie d'exploitation où les troupeaux étaient par conséquent de petite taille. Dans les conditions actuelles de la traite (encore très souvent manuelle) et de la monte, cette situation peut se prolonger pour des raisons pratiques. Dans les petits troupeaux, le contrôle laitier individuel peut, en effet, se faire plus facilement et avec plus de précision. En outre, dans ces petites unités qui n'utilisent en général qu'un seul bélier lors de la monte contrôlée, il ne se pose aucun problème d'isolement ou de tri des lots de lutte. De plus, les petits troupeaux utilisent uniquement de la main-d'œuvre familiale qu'il est plus facile d'intéresser aux différentes opérations de contrôle et de sélection que la main-d'œuvre rétribuée des grandes unités.

Cela n'exclut pas évidemment l'existence de quelques grands troupeaux de sélection dont le nombre est sans doute appelé à croître avec la généralisation de 1a traite mécanique. Ils sont, en fait, subdivisés en plusieurs unités d'accouplement de 60 à 80 brebis pendant la période de lutte contrôlée.

\section{C. - La taille des lots de lutte et l'effectif génétique utile}

On a vu que - essentiellement pour des raisons pratiques - les éleveurs des troupeaux inscrits n'employaient pour la monte contrôlée qu'un seul bélier par lutte. Il est donc normal que l'histogramme de la taille des lots de lutte se rapproche d'un histogramme log-normal au contraire de celui des nombres moyens de femelles allouées à chaque bélier dans les troupeaux non inscrits où les mâles sont mélangés aux brebis et où l'éleveur ajuste grossièrement leur nombre à celui des femelles à saillir (cf. fig. 3).

Un autre détail doit rester en mémoire pour l'analyse du phénomène : à partir d'une certaine date (un mois environ avant l'ouverture des laiteries), les agneaux nouvellement nés ne sont plus retenus pour l'élevage, qu'ils soient ou non issus de bélier inscrit. On peut préciser qu'on ne retient traditionnellement, en fait, pour la reproduction, que les agnelles déjà en âge d'être sevrées à ce moment-là, qui correspond au commencement de la traite. Tous les agneaux sont alors abattus comme agneaux de lait d'environ 7 à ro $\mathrm{kg}$ de poids vif. Ainsi, le choix des animaux de renouvellement se fait, aussi bien dans les troupeaux inscrits que non inscrits, sur une population tronquée. C'est-à-dire que, pour chaque année de lutte, une partie des brebis ne joue pas de rôle dans la sélection. Donc, la taille utile génétiquement des lots de femelles est certainement inférieure aux chiffres calculés dans le tableau 3 . Il n'est malheureusement possible que de donner approximativement l'ampleur de ce "tronquage", de 1'ordre de 25 p. IOO au moins (voir aussi BONELLI, I955).

\section{D. - Intervalle de génération}

Il est possible de l'estimer directement dans les troupeaux inscrits en calculant 1'âge moyen des reproducteurs à la mise bas. En excluant, bien entendu, les agnelles, on obtient 4,5 années pour le sexe femelle. Pour les mâles, le chiffre de 3,I ans est à retenir. Il s'agit, dans les deux cas, d'estimations qui ne distinguent pas les descendants par leur sexe.

La comparaison avec les troupeaux non inscrits qui ont des répartitions d'âge fort semblables après 3 ans (cf. tabl. 3), peut se faire à partir des seuls animaux 
de moins de 3 ans. On obtient alors pour les femelles des troupeaux inscrits et non inscrits des chiffres très voisins : 2,47 et $2,5 \mathrm{I}$ ans respectivement.

Chez les mâles, l'écart est plus net, ce qui résulte du fait que les mâles de l'année ne sont pratiquement pas utilisés dans les troupeaux inscrits, au contraire de ce qui semble se passer dans les troupeaux non inscrits. Avec 2,36 ans contre I,97 an, l'écart, qui sera en partie compensé par les mâles plus âgés, reste cependant minime.

BONELLI (I955) trouvait des chiffres avoisinants. Les valeurs de ces paramètres estimés à l'étranger (HOFMEYR et BOYAZOGLU, I968 et TURNER cité par ces derniers auteurs) sont également similaires.

\section{E. - Taux de renouvellement}

Le taux de renouvellement est évalué par le pourcentage d'agnelles qui est de I6,7 p. Ioo dans les troupeaux inscrits et 20,5 p. Ioo dans les troupeaux non inscrits. Ces chiffres, quoiqu'assez proches, sont significativement différents.

\section{F. - Migration des béliers}

Le tableau 5 et les figures 6 et 7 illustrent l'énorme importance prise par le centre de Barumini pour la fourniture des béliers à tous les troupeaux inscrits de Sardaigne. Cette fourniture est à sens unique : en I970 aucun bélier étranger au centre n'a été utilisé à Barumini (Fig. 6 ,b et 8 ) ; en outre, plus des $2 / 3$ des béliers utilisés dans les troupeaux de Barumini sont nés dans le même troupeau où ils sont utilisés.

Ce phénomène se répercute, atténué il est vrai, sur toute la population sarde, dans la mesure où les éleveurs non inscrits s'approvisionnent en béliers inscrits (tendance encouragée officiellement par des primes).

Cette prééminence des animaux du centre de Barumini s'explique, entre autres, par des conditions climatologiques très favorables, surtout pendant l'niver, et par la qualité exceptionnelle des pâturages naturels de la région située dans une zone de plaine et de collines; de plus, une partie des parcours est cultivée, permettant ainsi de mieux nourrir les animaux. La qualité des animaux de ce centre est, en outre, liée à la petite taille des troupeaux qui dépendent ainsi des soins attentifs d'un homme : le propriétaire lui-même. Le sevrage enfin est habituellement plus tardif. Ainsi a-t-on obtenu traditionnellement dans cette région des animaux très bien conformés, plus productifs parce que mieux nourris, donnant plus de lait aux contrôles et primés dans les concours. Enfin, le format moyen des animaux de cette région a toujours été réputée le mieux adapté pour toute l'île.

On se trouve donc en présence d'une population dans une situation pyramidale de sélection avec :

- Un centre (Barumini) de fournisseurs de la grande majorité de reproducteurs destinés aux troupeaux inscrits ; les éleveurs de Barumini n'utilisant eux-mêmes que des béliers de leurs propres élevages.

- D'autres centres de troupeaux inscrits qui ne sont en fait que des unités de multiplication, fournisseurs de reproducteurs pour les troupeaux non inscrits.

- Des troupeaux courants (non inscrits) qui forment la large plate-forme de la 
pyramide, n'utilisant pour leur part que des reproducteurs des centres définis ci-dessus ainsi que des béliers issus des troupeaux courants (en général ${ }_{₹}$ du même troupeau ou des troupeaux voisins).

Cette situation de hiérarchie entre les troupeaux inscrits pour la fourniture des reproducteurs utilisés en Sardaigne est très générale dans les races améliorées modernes. Classiquement admise dans les premières années de la constitution d'une nouvelle race (voir, par exemple, le rôle joué par les élevages du Simmental dans la création de la race bovine suisse tachetée rouge) une telle situation peut se perpétuer. WiEner l'observe dans les années cinquante aussi bien en race bovine Ayrshire (WiENER, I955) que dans de nombreuses races de moutons de Grande-Bretagne (WIENER, I961). Dans les troupeaux non inscrits, une telle tendance est beaucoup moins marquée avec in autorenouvellement inférieur $(34,5$ p. Ioo contre 57,6 p. I00) et une dispersion des échanges de mâles avec l'absence de la prédominance d'un centre comme celui de Barumini, mais cela ne fait finalement que retarder le phénomène.

Les inconvénients d'une telle politique d'élevage ont été dénoncés depuis longtemps ; cf., par exemple, ROBERTSON et ASKER (I95I). Rappelons-les brièvement : - L'effectif de 1a population génétiquement utile est restreint, avec augmentation de la consanguinité.

- Dans les trour zaux en vogue, trop sollicités, l'intensité de la sélection diminue.

- Par contrecoup, les possesseurs de nombreux troupeaux fort honorables, mais qui ne sont pas à la mode, perdent le goût pour un travail de sélection qui ne sera pas valorisé.

Tel qu'il est, le système actuel en place en Sardaigne ne présente toutefois pas que des inconvénients. Dans la me: are où les éleveurs n'utilisent pas les béliers de Barumini tout de même améliorés, ils auront en effet fortement tendance à utiliser leurs propres reproducteurs.

Sans vouloir brutalement modifier les habitudes établies, la meilleure politique actuelle est certainement d'encourager l'emploi progressif de béliers en provenance des différents autres centres d'élevage choisis d'après leur valeur génétique pour la production laitière. Le calcul d'index sur la descendance par comparaison des productions des filles du bélier à celle de ses contemporains du même élevage permet d'obtenir une classification objective de ces derniers indépendante en grande partie de l'action du milieu de production (POLY et al., I965). Un tel système entraînerait automatiquement une diversification des migrations de béliers entre élevages, minimisant les inconvénients signalés auparavant, accélérant l'amélioration génétique.

\section{CONCLUSIONS}

Les résultats de la présente étude illustrent sa nécessité sur le plan pratique et théorique.

En premier lieu, on s'aperçoit que la distribution de la taille génétique n'est pas forcément la même que celle de la taille apparente des troupeaux. On a pu voir également la stratification de la population et l'influence énorme prise actuellement par une petite partie de l'effectif (centre de Barumini) sur l'évolution génétique de 1a race, ainsi que les inconvénients de ce système. 
Pour l'étude des fréquences géniques comme nous l'avons ébauché (CAsu et al., r970) ou l'analyse de la consanguinité, il faudra bien sûr tenir compte de la situation que nous mettons en évidence

Reçu pour publication en août 1972.

\section{REMERCIEMENTS}

Le Dr G. WIEner de l'A. B. R. O. d'Edimbourg a eu la patience de lire en détail le manuscrit. Les auteurs lui doivent beaucoup, ainsi qu'à J. POLY et B. VISSAC pour leurs pertinentes critiques.

\section{SUMMARY}

\section{SOME DEMOGRAPHIC CHARACTERISTICS OF SARDINIAN SHEEP}

Almost all of the flocks registered in the Sardinian flock book were demographically analyzed ( 137 flocks out of I6I), and compared to a sample of 140 unregistered flocks.

The distribution of registered and unregistered flocks according to the number of females obeys a lognormal law.

In general, unregistered flocks had twice as many ewes as registered flocks ( 156.6 against 85.2).

The average number of females per ram, which are liable to have breeding progeny, is twice as great in registered flocks as in unregistered flocks ( 73 females in one case, $3^{2}$ in the other).

The generation interval, estimated by the average age of breeding animals at the birth of their progeny, is 3 .I years for males and 4.5 for females in registered flocks, grouping the sexes of the progeny. A comparison with unregistered flocks, based on animals of less than three years, shows great similarity for the females. As to the males in unregistered flocks, they have a slightly reduced generation interval.

The rate of annual renewal of females is $17 \mathrm{p}$. Ioo in registered flocks and $20 \mathrm{p}$. Ioo in unregistered flocks.

A study of ram migration shows that the Sardinian population presents a pyramidal selection sitructure with the Barumini center, which furnishes the majority of non-aboriginal rams used in registered flocks, at the apex. The center receives no gene from the exterior. Such a hierarchial structure, although rather common in modern improved cattle and sheep breeds, should be transitory, if the efficiency of selection is to be increased by enlarging its bases.

\section{RÉFÉRENCES BIBLIOGRAPHIQUES}

Anonyme 1969. Enquête communautaire sur la structure des exploitations agricoles en 1967. Statistiques agricoles Suppl., Série Études $n^{\circ} 42$ et 42 bis. Ministère de l'Agriculture, Paris.

Bettini T. M., 1952. Su alcune cause di variazione della lunghezza della lattazione della produzione lattea nella pecora Sarda. Riv. Zootec., 25, 3-ro.

Bonelli P., I955. La pecora Sarda alla luce dell'indagine statistica. Ital. Agric., 92, 49 I-503.

Bonelli P., i962. La selezione della pecora Sarda. Prog. Agric., 8 (10), 7 p.

CASU S., I97r. Allevamento ovino da latte in Sardegna : situazione attuale e possibilità di evoluzione. Options méditerranéennes (7), I00-106.

Casu S., Boyazoglu J. G., Lauvergne J. J., 197o. Hérédité des pendeloques dans la race ovine sarde. Ann. Gênét. Sél. anim., 2, 249-26r. 
Hofmeyr, J. H. Boyazoglu J. G., I968. Preliminary notes on vital statistics of and selection in the merino sheep industry of South Africa. Proc. III Cong. South Afr. Genetic Soc., july, 1966, 75-77.

Inda L., 1969. Aspetti economici della trasformazione del latte di pecora in Sardegna Giornate di studio sui problemi della trasformazione del latte di pecora in Sardegna. Istituto zootecnico et caseario per la Sardegna, Sassari.

Passino F., r936. Le vicende dell'allevamento della pecora sarda. Nuovi Ann. agricolt., 16, 253-298

Poly J., Poutous M., Frebling J., r965. Méthode de calculs d'index de production laitière. Bull. tech. Inf. Ing. Serv, agr. (205), 964-997.

Robertson A., Asker A. A., 195 I. The genetic history and breed structure of British Friesian cattle. Empire J. Exper. Agric., 19, II 3 -1 30.

Wiener G., r955. Migration of pedigree Ayrshire cattle in Great-Britain. J. Agric. Sci., 45, 476-48o.

Wiener G., I96r. Population dynamics in fourteen lowland breeds of sheep in Great-Britain. J.Agric. Sci., 57, 2 I-28. 